\title{
Predictive minds in Ouija board sessions
}

\author{
Marc Andersen ${ }^{1,2,3}$ (D) Kristoffer L. Nielbo ${ }^{2,3,4}$. \\ Uffe Schjoedt ${ }^{1,2,3}$. Thies Pfeiffer ${ }^{5,6}$. \\ Andreas Roepstorff ${ }^{1,3}$. Jesper Sørensen ${ }^{1,2,3}$
}

\begin{abstract}
Ouija board sessions are illustrious examples of how subjective feelings of control - the Sense of Agency (SoA) - can be manipulated in real life settings. We present findings from a field experiment at a paranormal conference, where Ouija enthusiasts were equipped with eye trackers while using the Ouija board. Our results show that participants have a significantly lower probability at visually predicting letters in a Ouija board session compared to a condition in which they are instructed to deliberately spell out words with the Ouija board planchette. Our results also show
\end{abstract}

\section{Marc Andersen}

mana@ cas.au.dk

Kristoffer L. Nielbo

knielbo@sdu.dk

Uffe Schjoedt

us@cas.au.dk

Thies Pfeiffer

thies.pfeiffer@uni-bielefeld.de

Andreas Roepstorff

andreas.roepstorff@ cas.au.dk

Jesper Sørensen

jsn@cas.au.dk

1 School of Culture and Society, Aarhus University, Aarhus, Denmark

2 Religion, Cognition and Culture, Aarhus University, Aarhus, Denmark

3 Interacting Minds Centre, Aarhus University, Aarhus, Denmark

4 Department of History, University of Southern Denmark, Odense, Denmark

5 Technical Faculty, Bielefeld University, Bielefeld, Germany

6 Cognitive Interaction Technology Center of Excellence (CITEC), Bielefeld University, Bielefeld, Germany 
that Ouija board believers report lower SoA compared to sceptic participants. These results support previous research which claim that low sense of agency is caused by a combination of retrospective inference and an inhibition of predictive processes. Our results show that users in Ouija board sessions become increasingly better at predicting letters as responses unfold over time, and that meaningful responses from the Ouija board can only be accounted for when considering interactions that goes on at the participant pair level. These results suggest that meaningful responses from the Ouija board may be an emergent property of interacting and predicting minds that increasingly impose structure on initially random events in Ouija sessions.

Keywords Sense of agency. Ouija board - Predictive minds · Eye tracking - Religious and paranormal belief

\section{Introduction}

Sense of agency (SoA) refers to the feeling of generating and controlling actions in order to influence external events (Haggard and Tsakiris 2009; Haggard and Chambon 2012; Moore and Fletcher 2012; Moore and Obhi 2012). SoA is essential to ideas of responsibility, and plays a pivotal role in justice systems, where the accused's awareness of having committed a criminal act is of great importance for sentencing (Haggard and Chambon 2012). SoA also seems to play an important role in many mental illnesses. In schizophrenia, for example, patients will sometimes report that their actions result from control by some external entity or person (Blakemore et al. 2002), and in depression, patients often report feelings of helplessness and loss of agency (Haggard and Chambon 2012). Researchers have frequently gestured toward Ouija boards sessions to exemplify how SoA can be convincingly manipulated to the extent that participants will spell out words with a planchette but collectively claim that an invisible third party is actually producing the movements (e.g. Moore and Fletcher 2012; Wegner and Wheatley 1999; Carruthers 2010; Coyle et al. 2012; Dewey and Carr 2013; Olson et al. 2016). Despite frequent references to the Ouija board phenomenon, however, there is a striking absence of empirical studies investigating SoA in real life Ouija board sessions.

The Ouija board is normally used by two or more participants who place their hands lightly on top of a triangular planchette. The planchette is then moved around a board with the alphabet, numbers from 0 to 9, and the words "YES", "NO" and "GOODBYE" printed on it. Typically, participants will ask the board a question, to which the board will often supply with a coherent and meaningful 'response' (Wegner 2002). Responses are usually believed to issue from supernatural agents; in many cases these are deceased relatives or former residents of the area where the Ouija session is taking place. Importantly, such beliefs are facilitated by participants' subjective experience of not moving the planchette themselves, i.e. their diminished SoA.

One influential theory claims that SoA mainly arises from processes that serve motor control (Blakemore et al. 2002). According to this account, the brain predicts the sensory consequences of an action, and then compares this prediction to the actual consequences. When prediction and consequence match, the result is the feeling that "I did that". Mismatches, however, precipitate a decrease in experienced SoA (Haggard 
and Chambon 2012; Blakemore et al. 2002). In Ouija boards sessions, the low SoA reported by participants may therefore be caused by difficulty predicting the board's responses. This, however, seems like a paradox: how can participants on the one hand produce coherent and meaningful responses through the board while on the other hand be unable to predict those very same responses?

The Ouija board phenomenon is often explained by analogy to facilitated communication (FC) (Burgess et al. 1998; Wegner et al. 2003). FC is a now discredited technique that was claimed to help communicatively-impaired clients to communicate through a human 'facilitator'. In FC, the facilitator will typically hold the hands of the impaired client and try to detect what the client is trying to communicate on a keyboard or spelling board. Several studies have shown, however, that the facilitators, unbeknownst to themselves, are really doing the communicating (e.g. Wegner et al. 2003; Cabay 1994). In a particularly renowned example, Wheeler and colleagues gave separate questions through headphones to clients and facilitators, and the answers turned out to match the questions posed to the facilitators (Wheeler et al. 1993). Because of obvious structural and functional similarities between Ouija board sessions and FC, these phenomena have at times been collectively explained by proponents of a 'retrospective' account of SoA in which a general-purpose mechanism retrospectively infers the causal origins of actions and their effects by relying primarily on external, situational cues and beliefs about the situation (Wegner 2002; Wegner et al. 2003). This account argues that beliefs about the Ouija board and FC are pivotal in determining SoA in participants. However, while evidence suggests that both predictive and retrospective processes are important for SoA (Moore and Fletcher 2012; Moore and Obhi 2012), it remains unclear from both accounts how meaningful responses emerge in the contexts of FC and Ouija sessions.

In what follows, we present data from the first field study on predictive behaviour and SoA. In this exploratory study, we used mobile eye tracking technology to compare predictive gaze behaviour in Ouija Board sessions with a voluntary action condition in which participants in pairs were asked to voluntarily spell words on the board with the planchette. Because we assume that vision is crucially involved in the construction of meaningful responses from the Ouija board (albeit potentially unbeknownst to the participants themselves), we operationalised predictive behaviour through manual annotation of participants' gaze data. Similarly, we operationalised motor outcome by manually annotating responses from the Ouija board (i.e. which letters the planchette would point to). Finally, we investigated the effects of belief on SoA through the administration of a questionnaire, asking questions about participants' belief in the Ouija board and their SoA during the Ouija board sessions.

\section{Methods}

\subsection{Ethics and informed consent}

The methods in the following study were carried out in accordance with all relevant guidelines and regulations issued by the National Committee on Health Research Ethics, Denmark. Informed consent was obtained from all participants. 


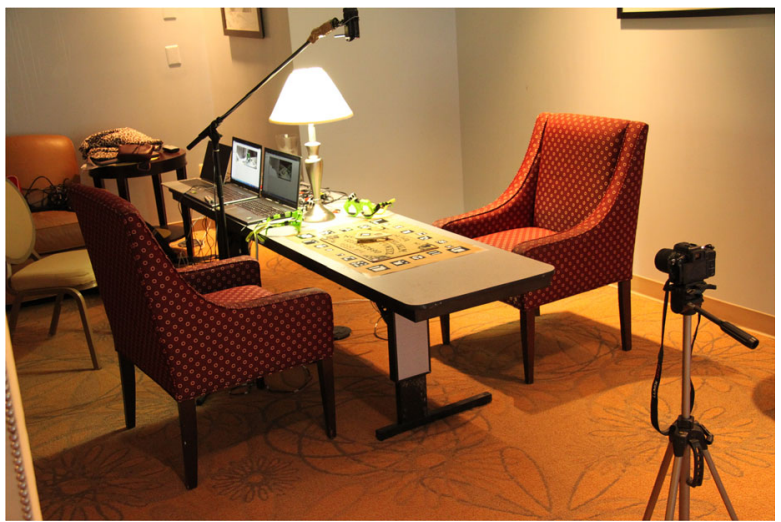

Fig. 1 The experimental setup

\subsection{Participants}

Forty American volunteers ( 25 females; age: $\mathrm{M}=42.5, \mathrm{SD}=9.7$, Range $=25-67$ ) were recruited in pairs on site at a Ouija board conference. The majority of participants had experience with using the Ouija Boards ("Do you consider yourself an experienced Ouija Board user", 5-point Likert scale, -2 to +2 , Mean $=0.7, \mathrm{SD}=1.2$ ). Twenty-eight participants owned a Ouija board themselves. The sample size was based on the number of participants that were willing to participate in the study during the conference.

\subsection{Procedure}

Sitting in front of each other with a Ouija board on a table between them (see Fig. 1), the participants were informed about the experimental procedure and equipped with mobile eye trackers which were then calibrated (see 2.4 Equipment).

Participants went through two conditions, ${ }^{1}$ here referred to as the 'voluntary action condition' and the 'Ouija condition' In the 'Ouija condition' participants were instructed to use the Ouija board as they normally would, and participants were free to ask the Ouija Board anything they liked. Questions often resulted in the Ouija board 'responding' to the participants. The 'Ouija condition' lasted approximately $10 \mathrm{~min}$, after which the participants were asked to stop. In the 'voluntary action condition' participants were asked to deliberately move the planchette to 'YES', deliberately move

\footnotetext{
${ }^{1}$ Participants also went through a third condition which is not reported in the present article. Briefly, participants repeated five predetermined questions from the experimenter. Four questions invited YES/NO responses from the board, one invited an open-ended response. Only four pairs produced a meaningful message in the open-ended task. Further, it became clear to us that participants needed not rely on vision to locate "YES"/"NO", and therefore this condition could not be used for the purpose of the study. Importantly, while Ouija board users may move the planchette to "YES" and "NO" without looking, spelling words using the alphabet on the Ouija board, requires visual guidance. This is mainly because "YES" and "NO" are both spatially isolated far from the letters and numbers on the Ouija board, but also because responses of "YES" and "NO" will generally be accepted as such even in cases where the planchette is in the near vicinity of "YES" and "NO" and not directly on them. For the reasons above, we did conduct any data analysis on this condition.
} 
the planchette to 'NO', and deliberately use the planchette to spell 'BALTIMORE'. The order of the sessions was counterbalanced.

Following the experimental session participants were asked to fill in a questionnaire on demographics and experiences in the Ouija condition. Items included experienced sense of agency; amount of push they felt from the other player; the degree to which they deliberately pushed the planchette; and experience of a supernatural entity. Participants were asked about their beliefs in the Ouija board, their previous experience with Ouija boards, their impression of their partner, and their levels of religiosity and spirituality. Most questionnaire items were included for exploratory purposes and was not included in the final data analysis (but see 2.7 Data Availability).

\subsection{Equipment}

Two low-cost (self-made) mobile eye trackers (6IR940nm eye camera, 520TVL 940 nM LED scene camera) were used to record participants' eye movements during Ouija board sessions. Tracking was done using the open source software Haytham (Mardanbegi 2009) on Fujitsu E754 Lifebooks with Windows 7. This system provided a monocular sampling procedure at a $720 \times 480$ pixel resolution with a sampling rate of $15 \mathrm{~Hz}$ for the eye tracking. A single user controlled calibration procedure with 4 calibration points was used. Finally, an overview camera was placed approximately $1 \mathrm{~m}$ above the Ouija board.

\subsection{Eye movement data \& manual annotation}

For each pair of participants, eye gaze recordings of both participants were synchronised with video footage from the overview camera. In this way, a video containing eye gaze from both participants and their interaction with the Ouija board was produced for each condition (see Fig. 2).

Two independent blind coders manually annotated the videos in the annotation software ELAN (Lausberg and Sloetjes 2009). In the 'Voluntary action condition', the coders annotated time periods in which the experimenter instructed participants to deliberately move the planchette (on sentence level), and time periods when participants deliberately moved the planchette as instructed by the experimenter (on sentence level and letter level). In the 'Ouija Condition', the coders annotated time periods in which participants posed questions to the Ouija board (on sentence level) and time periods of the responses from the Ouija board (on sentence level and letter level). In both conditions, time periods were annotated in which participants (1) looked at the planchette and (2) looked at the upcoming letter (prediction). With respect to the latter, coders were instructed that focal gaze had to fall on the exact letter that the planchette would subsequently reach. Any amount of time looking on the exact letter would qualify as a prediction. On average, it took around one and a half hours for the coders to annotate $1 \mathrm{~min}$ of video. The coders manually annotated $3-4 \mathrm{~h}$ of video in total.

A data set was constructed from the annotations in order to explore eye gaze within and across conditions. In this data set, participant eye behaviour in each condition was split into a series of discrete responses on the board, so that each response could be separately analysed. This allowed us to evaluate the data quality of eye movements in each response as opposed to the data quality of an entire condition. For each response, 


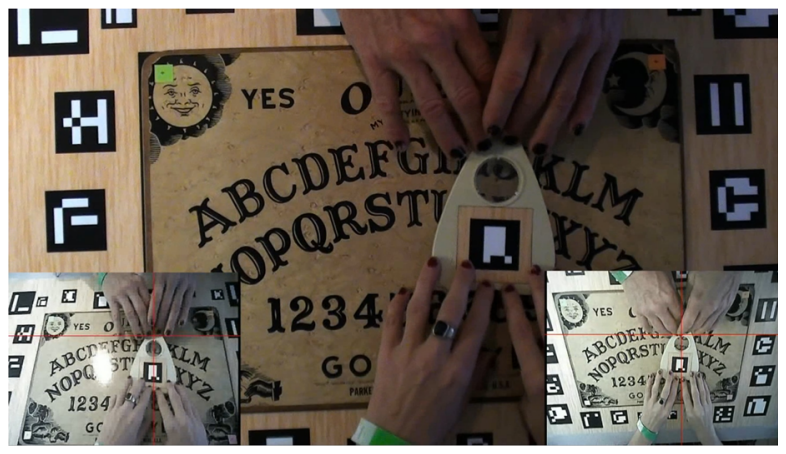

Fig. 2 Example of video for coders

it was noted whether or not participants predicted each individual letter. In addition to coding this individual eye behaviour, we also added a 'pair level' to the data frame, where we coded whether or not at least one of the participants in the pair predicted the letters in the responses. This allowed us to compare the ability to predict letters in individuals seen in isolation with pairs of participants.

\subsection{Data anlysis}

Recordings from one pair in the 'Ouija board session' and one pair in the 'Voluntary action condition' were lost due to technical issues. Before conducting any analyses, we removed all responses with only 1 semantic unit from the data after learning that Ouija board users do not need to rely on their vision to locate "YES", "NO", and "GOODBYE". Responses with poor data quality ${ }^{2}$ (below 60\%) were removed.

This left us with a total of 176 responses suitable for statistical analysis (data quality (DQ): $\mathrm{M}=83.7 \%, \mathrm{SD}=11.5 \%$ ). Of these, 33 responses were from the 'Voluntary action session', where participants were instructed to deliberately spell the word "BALTIMORE" (DQ: $\mathrm{M}=83.0 \%, \mathrm{SD}=11.5 \%$ ), and 143 responses were from the 'Ouija condition', where participants interacted with the board in a natural manner (DQ: $87.3 \%, \mathrm{SD}=11.7 \%$ ). Out of the 143 responses in the 'Ouija condition', 67 responses were meaningful (e.g. Participant: "Would you like to spell your first name?", Ouija board: "HENRY") and 76 responses were not meaningful (e.g. Participant: "Where did you pass away?", Ouija Board: "STACW").

To test the hypothesis that low sense of agency in Ouija Board Sessions results from difficulty predicting the responses 'picked' by the planchette, we performed a mixed effects logistic regression with 'predicts upcoming letter' as outcome and 'condition' as a fixed effect (M1). Because a significant difference between conditions in the ability to predict letters could be driven by non-meaningful Ouija board responses, we also performed the same analysis with only meaningful responses from the Ouija board (M1b).

To test the hypothesis that low SoA is related to participants' beliefs about the Ouija board, we performed a bivariate analysis of 6 questionnaire items reported by the 40

\footnotetext{
${ }^{2}$ Data quality was obtained through a software that estimated the percentage of time during a response where the participant's eye gaze was captured by the eye tracker (i.e. the percentage of time that the horizontal and vertical red line was present in the eye tracking videos).
} 
participants. Two of these items were questions related to the participants' belief about the Ouija board and four of these items where related to whom or what the participants attributed the planchette's movement. We corrected for multiple comparisons using a Bonferroni-Holm test (Holm 1979).

To explore how participants in each condition predicted letters over time in meaningful responses, we performed a mixed effects logistic regression, with 'predicts upcoming letter' as outcome and 'condition', 'letter number' and 'individual level /pair level' as fixed effects (M2).

In all the mixed effect models we used a maximal random structure with participants nested within pairs as intercept and the fixed effects as random slopes. Due to convergence issues we removed pairs from the random structure in two models (M1b \& M2), and 'letter number' and 'individual level /pair level' from one model (M2). In case of significant interactions, we performed post hoc tests. Visual inspection of residual plots revealed only minimal or no obvious deviations from homoscedasticity or normality. To perform the analyses, we used lmerTest 2.0-30 (Kuznetsova et al. 2016) 'psych' 1.6.8, (Revelle 2016), and R 3.2.4 (R Core Team 2016).

\subsection{Data availability}

The data generated and analyzed during the current study are available through the Open Science Framework (link: https://osf.io/hcb83/) ([Dataset] Andersen et al. 2017).

\section{Results}

Results revealed a significant effect of the 'Ouija condition', indicating that participants had a 37.9\% lower chance of predicting each letter in the 'Ouija condition' compared to the 'Voluntary action condition' $\left(\beta=-1.88, \mathrm{SE}=0.224, p<0.001, \mathrm{R}^{2} \mathrm{~m}=0.17, \mathrm{R}^{2} \mathrm{c}=\right.$ 0.34 ). Because this difference between conditions in the ability to predict letters could be driven by non-meaningful Ouija board responses, which intuitively would seem harder to predict than meaningful responses, we also performed the same analysis with only meaningful responses from the Ouija board. Looking only at meaningful responses, results still revealed a significant effect of the 'Ouija condition', indicating that participants had a $21.3 \%$ lower chance of predicting each letter in the 'Ouija condition' compared to the 'Voluntary action condition' $(\beta=-1.19, \mathrm{SE}=0.347$, $p<0.001, \mathrm{R}^{2} \mathrm{~m}=0.09, \mathrm{R}^{2} \mathrm{c}=0.19$ ).

Results revealed significant correlations between all questionnaire items. Generally, believers reported that the planchette moved by itself; that the other participant did not push the planchette; that they themselves did not push the planchette; and that they experienced contact with a supernatural agent (see Table 1). Conversely, sceptics in general reported that the planchette did not move by itself; that the other participant pushed the planchette; that they themselves, although to a lesser extent, also pushed the planchette; and that they did not experience contact with a supernatural being (see Table 1).

In our holistic model, where we looked at the effects of condition, letter number and individual level vs. pair level on the ability to predict upcoming letters, results revealed a significant effect of the 'Ouija Condition' indicating that participants had a $38.0 \%$ 
Table 1 Correlations between questionnaire items

\begin{tabular}{|c|c|c|c|c|}
\hline & $\begin{array}{l}\text { Did you feel as if } \\
\text { the planchette } \\
\text { moved on its own } \\
\text { during the } \\
\text { session? } \\
\text { (9-point Likert } \\
\text { scale) }\end{array}$ & $\begin{array}{l}\text { Did you feel } \\
\text { as if the other } \\
\text { participant } \\
\text { deliberately } \\
\text { pushed } \\
\text { the planchette? } \\
\text { (9-point Likert } \\
\text { scale) }\end{array}$ & $\begin{array}{l}\text { Did you } \\
\text { deliberately } \\
\text { push the } \\
\text { planchette? } \\
\text { (9-point Likert } \\
\text { scale) }\end{array}$ & $\begin{array}{l}\text { Did it feel as if you } \\
\text { made contact with } \\
\text { an entity other than } \\
\text { the persons involved } \\
\text { in the experiment } \\
\text { (e.g. a spirit, a ghost, } \\
\text { a demon, an alien, } \\
\text { etc.)? } \\
\text { (9-point Likert scale) }\end{array}$ \\
\hline $\begin{array}{l}\text { The Ouija board can be } \\
\text { used to contact entities } \\
\text { such as spirits, ghost, } \\
\text { demons, angels etc. } \\
\text { (5-point Likert scale) }\end{array}$ & $\begin{array}{l}r=0.526 \\
p<0.001\end{array}$ & $\begin{array}{l}r=-0.423 \\
p=0.007\end{array}$ & $\begin{array}{l}r=-0.463 \\
p=0.003\end{array}$ & $\begin{array}{l}r=0.638 \\
p<0.001\end{array}$ \\
\hline $\begin{array}{l}\text { The Ouija board is really } \\
\text { driven by the } \\
\text { subconscious } \\
\text { mind of its users (i.e. } \\
\text { the ideomotor effect) } \\
\text { (5-point Likert scale) }\end{array}$ & $\begin{array}{l}r=-0.481 \\
p=0.002\end{array}$ & $\begin{array}{l}r=0.442 \\
p=0.004\end{array}$ & $\begin{array}{l}r=0.339 \\
p=0.033\end{array}$ & $\begin{array}{l}r=-0.658 \\
\mathrm{p}<0.001\end{array}$ \\
\hline
\end{tabular}

lower chance of predicting letters in the 'Ouija condition' compared with the 'Voluntary action condition' ( $\beta=-1.655, \mathrm{SE}=0.411, p<0.001)$. Furthermore, there was a significant effect of pair level, indicating that there was a $15.5 \%$ higher chance in the 'Voluntary action condition' and a $31.0 \%$ higher chance in the 'Ouija condition' that at least one of the participants in a pair would predict upcoming letters compared to a single participant seen in isolation $(\beta=1.293, \mathrm{SE}=0.148, \mathrm{p}<0.001)$. In other words, there was a significant higher chance that at least one out of two participants in a pair would predict upcoming letters compared to a single participant seen in isolation, which in not surprising. Finally, we found a significant interaction between 'letter number' and 'Ouija condition', indicating that participants in the 'Ouija condition' became better at predicting upcoming letters as a response from the board unfolded over time $(\beta=-0.180, \mathrm{SE}=0.081, p=0.026)$. In other words, while participants exhibited a stable ability to predict upcoming letters in the 'Voluntary action condition', their ability to predict upcoming letters in the 'Ouija board condition' steadily became better and better. Post hoc test revealed that on average, participants' chance of predicting upcoming letters in the 'Ouija Condition' increased by roughly 5\% by each consecutive letter in the meaningful responses (See Fig. 3). The model explained 17.9\% of the variance in the data without the random factors $\left(\mathrm{R}^{2} \mathrm{~m}\right)$ and $29.6 \%$ of the variance with the random factors $\left(\mathrm{R}^{2} \mathrm{c}\right)$.

\section{Discussion}

For the first time in a natural setting, we provide evidence for why Ouija board users exhibit a reduced Sense of Agency. In our field experiment, participant pairs were equipped with mobile eye trackers and went through two conditions: 1) the 'Voluntary 


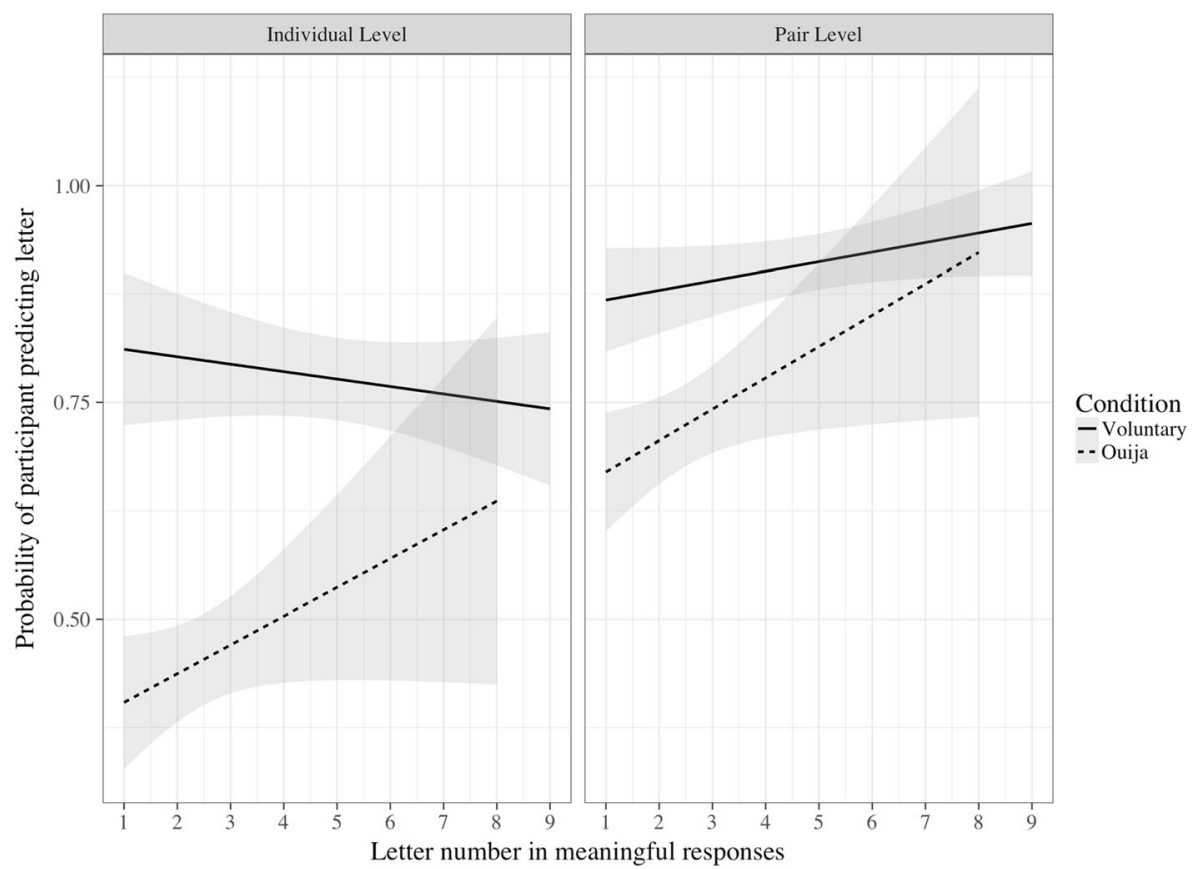

Fig. 3 Probability of predicting letters in meaningful responses as a function of letter number on individual and pair level

action condition' in which participants were asked to deliberately make responses on the Ouija board and 2) the 'Ouija condition' in which participants were asked to use the Ouija board as they normally would see fit. Participants then filled out a questionnaire about their experiences in the Ouija condition. Our paradigm operationalised predictive behaviour through eye gaze and participants' belief about the board through questionnaire items. This allowed us to explore theoretical accounts which suggest that both predictive and retrospective processes are important with respect to SoA (Moore and Fletcher 2012). When looking at the predictive gaze behaviour of individual participants, participants in the 'Ouija board condition' exhibited significant difficulties in predicting which letters would be 'picked' by the planchette compared to the 'Voluntary action condition' (see Fig. 3). This is also true when we only consider meaningful responses, where participants logically would have to predict letters in order to spell the response. In other words, the decreased ability to predict letters in the 'Ouija condition' cannot be fully accounted for by non-meaningful responses which are likely harder to predict. Overall, these findings are in accord with predictive accounts of SoA, in which the low SoA in Ouija board sessions arises from a mismatch between the predicted and actual sensory consequences of an action (Haggard and Chambon 2012; Blakemore et al. 2002).

We found a strong relationship between beliefs about the Ouija board and the reported experience of the 'Ouija condition'. Participants who agreed with the statement, "The Ouija board can be used to contact entities such as spirits, ghost, demons, angels etc.", typically also agreed that it felt as if the planchette moved on its own; that neither they nor the other participants pushed the planchette; and that contact was made 
with a supernatural agent. Conversely, participants who agreed with the statement, "The Ouija board is really driven by the subconscious mind of its users (i.e. the ideomotor effect)", typically reported the opposite, namely that it did not feel as if the planchette moved on its own; that the other participant and, albeit to a lesser degree, they themselves pushed the planchette deliberately; and that no contact was made with a supernatural agent. Although our bivariate analysis cannot be used to infer causality, these finding are nevertheless in accord with a retrospective account of SoA, in which a general-purpose mechanism retrospectively infers the causal origins of actions and their effects by relying primarily on external, situational cues and beliefs about the situation (Wegner 2002; Wegner et al. 2003). In sum, our results accord with recent research which suggests that both predictive and retrospective processes are important aspects of SoA (Moore and Fletcher 2012).

Overall, it appears that participants in the 'Ouija condition' generally underestimate their own contribution to the joint interaction. While those who believe in the possibility that the Ouija board can be used to communicate with supernatural agents to a very large degree attribute agency to something outside the immediate dyad, participants who believe in the opposite generally report that the other player pushed the planchette more than they did themselves. This finding accords with recent work on force escalation. In an experiment, Shergill et al. (2003) instructed participants in pairs to alternately apply pressure though a force transducer on each other's hands. Although participants were instructed to apply the same amount of pressure as had just been exerted on them, results showed that the forces applied rapidly escalated. As such, these findings suggest that self-generated forces are generally perceived as weaker than external forces of the same magnitude (Shergill et al. 2003).

As in the case of Shergill and colleagues, some patterns and dynamics first become visible when looking at how pairs of participants perform as opposed to individuals in isolation. In our study, in addition to coding if participants seen in isolation predicted letters in responses, we also coded predictions on pair level, to see if at minimum one of the two participants in a pair predicted letters in responses. Individuals seen in isolation have a much lower probability on average of predicting the letters in the 'Ouija condition' compared to the 'voluntary action condition' (Fig. 3). However, when we look at the pair level, we see that pairs in the 'Ouija condition' on average predict the letters of meaningful responses as well as isolated individuals do when purposely spelling responses in the 'voluntary action condition'. In other words, a pair that moves the Ouija planchette in a predominantly non-deliberate way collectively predict letters as well as an individual seen in isolation that is moving the planchette on purpose.

While the ability to predict letters remains stable over time on an individual and pairbased level in the 'voluntary action condition', this is not the case in the 'Ouija condition'. Although individual participants do not at any point seem to predict letters as well in this condition as the deliberately produced responses in the 'Voluntary action condition', individuals in the 'Ouija condition' do steadily get better at predicting the letters as meaningful responses unfold over time. The same is true when looking at the pair level in this condition; while pairs in the 'Ouija condition' are less able to predict the beginnings of meaningful responses from the board compared to individuals in the 'Voluntary action condition', they steadily become better at predicting to the point where, in many cases, they are able to predict letters as well as pairs in the 'voluntary action condition' (see Fig. 3). 
Our study suggests that successful Ouija board sessions critically depend on joint action. It also suggests an answer to the apparent paradox of how Ouija users on the one hand can exhibit difficulties in predicting letters in responses, while on the other hand are assumed to produce those very same responses themselves. It seems that meaningful responses from the Ouija board are an emergent property of interacting predictive minds that increasingly impose structure on initially random events in the sessions. While early letters in a meaningful Ouija board response appear to occur at random, meaningful word options available to the participants decrease as the response from the Ouija board unfolds. This, in turn, makes it easier for a pair of participants to collectively predict (and unconsciously construct) the responses from the Ouija board. These findings are compatible with research on divinatory practices that claim that such practices are universally characterised by humans imposing meaning and structure on random events (Morgan 2016). Our results add to this by showcasing just how predictive minds in interaction achieve the feat of creating meaningful sensations of external agency.

Acknowledgements We gratefully acknowledge the great amount of help and assistance in facilitating data collection from Robert Murch, chairman of the Talking Boards Historical Society. We would also like to extend our thanks to Sissel Louise Jørgensen and Cecilie Cedergreen for manually annotating large amounts of gaze data, and to Cecilie Cedergreen for her additional assistance during the field experiment.

Author contributions M. Andersen developed the concept of the study. All authors contributed to the study design. M. Andersen build the eye-tracking equipment and collected the data. T. Pfeiffer wrote software for data quality extraction. K.L. Neilbo and M. Andersen analysed the data. M. Andersen drafted the manuscript and U. Schjoedt, A. Roepstorff, and J. Sørensen provided critical revisions.

Funding This project has been supported by seed funding from the Interacting Minds Centre at Aarhus University.

\section{Compliance with ethical standards}

Competing financial interests The authors declare no competing financial interests.

Open Access This article is distributed under the terms of the Creative Commons Attribution 4.0 International License (http://creativecommons.org/licenses/by/4.0/), which permits unrestricted use, distribution, and reproduction in any medium, provided you give appropriate credit to the original author(s) and the source, provide a link to the Creative Commons license, and indicate if changes were made.

\section{References}

[Dataset] Andersen, M., Nielbo, K. L., Schjoedt, U., Pfeiffer, T., Roepstorff, A. \& Sørensen, J. (2017). OuijaCon Dataset, Open Science Framework (https://osf.io/hcb83/). https://doi.org/10.17605/OSF. $\mathrm{IO} / \mathrm{HCB} 83$

Blakemore, S. J., Wolpert, D. M., \& Frith, C. D. (2002). Abnormalities in the awareness of action. Trends in Cognitive Sciences, 6(6), 237-242.

Burgess, C. A., Kirsch, I., Shane, H., Niederauer, K. L., Graham, S. M., \& Bacon, A. (1998). Facilitated communication as an ideomotor response. Psychological Science, 9(1), 71-74. 
Cabay, M. (1994). Brief report: a controlled evaluation of facilitated communication using open-ended and fill-in questions. Journal of Autism and Developmental Disorders, 24(4), 517-527.

Carruthers, G. (2010). A problem for Wegner and colleagues' model of the sense of agency. Phenomenology and the Cognitive Sciences, 9(3), 341-357.

Coyle, D., Moore, J., Kristensson, P. O., Fletcher, P., \& Blackwell, A. (2012). I did that! Measuring users' experience of agency in their own actions. Proceedings of the SIGCHI Conference on Human Factors in Computing Systems (pp. 2025-2034).

Dewey, J. A., \& Carr, T. H. (2013). When dyads act in parallel, a sense of agency for the auditory consequences depends on the order of the actions. Consciousness and Cognition, 22(1), 155-166.

Haggard, P., \& Chambon, V. (2012). Sense of agency. Current Biology, 22(10), R390-R392.

Haggard, P., \& Tsakiris, M. (2009). The experience of agency: feelings, judgments, and responsibility. Current Directions in Psychological Science, 18(4), 242-246.

Holm, S. (1979). A simple sequentially rejective multiple test procedure. Scandinavian Journal of Statistics, $65-70$.

Kuznetsova, A., Brockhoff, P.B., \& Christensen, R. H. B. (2016). lmerTest: Tests in linear mixed effects models. R package version 2.0-30. https:/CRAN.R-project.org/package=lmerTest

Lausberg, H., \& Sloetjes, H. (2009). Coding gestural behavior with the NEUROGES-ELAN system. Behavior Research Methods, Instruments, \& Computers, 41(3), 841-849 URL: http://tla.mpi.nl/tools/tla-tools/elan/.

Mardanbegi, D. (2009). Haytham gaze tracker. (https://sourceforge.net/projects/haytham/?source=navbar).

Moore, J. W., \& Fletcher, P. C. (2012). Sense of agency in health and disease: a review of cue integration approaches. Consciousness and Cognition, 21(1), 59-68.

Moore, J. W., \& Obhi, S. S. (2012). Intentional binding and the sense of agency: a review. Consciousness and Cognition, 21(1), 546-561.

Morgan, D. (2016). Divination, material culture, and chance. Material Religion, 12(4), 502-504.

Olson, J. A., Landry, M., Appourchaux, K., \& Raz, A. (2016). Simulated thought insertion: influencing the sense of agency using deception and magic. Consciousness and Cognition, 43, 11-26.

R Core Team. (2016). R: A language and environment for statistical computing. R Foundation for Statistical Computing, Vienna, Austria. URL https://www.R-project.org/.

Revelle, W. (2016) psych: Procedures for Personality and Psychological Research, Northwestern University, Evanston, Illinois, USA, https://CRAN.R-project.org/package=psych Version = 1.6.12.

Shergill, S. S., Bays, P. M., Frith, C. D., \& Wolpert, D. M. (2003). Two eyes for an eye: the neuroscience of force escalation. Science, 301(5630), 187-187.

Wegner, D. M. (2002). The illusion of conscious will. Cambridge: MITPress.

Wegner, D. M., \& Wheatley, T. (1999). Apparent mental causation: sources of the experience of will. American Psychologist, 54(7), 480-492.

Wegner, D. M., Fuller, V. A., \& Sparrow, B. (2003). Clever hands: uncontrolled intelligence in facilitated communication. Journal of Personality and Social Psychology, 85(1), 5-19.

Wheeler, D. L., Jacobson, J. W., Paglieri, R. A., \& Schwartz, A. A. (1993). An experimental assessment of facilitated communication. Mental Retardation, 31(1), 49-59. 